\title{
Design and Analysis of the International X-Ray Observatory Mirror Modules
}

\author{
Ryan S. McClelland*a, Timothy M. Carnahan ${ }^{\mathrm{b}}$, David W. Robinson ${ }^{\mathrm{b}}$, Timo T. Saha ${ }^{\mathrm{b}}$ \\ ${ }^{a}$ SGT Inc. 7701 Greenbelt Road, Suite 400, Greenbelt, Maryland 20770, USA \\ bASA Goddard Space Flight Center, Greenbelt, MD USA 20771, USA
}

\begin{abstract}
The Soft X-Ray Telescope (SXT) modules are the fundamental focusing assemblies on NASA's next major X-ray telescope mission, the International X-Ray Observatory (IXO). The preliminary design and analysis of these assemblies has been completed, addressing the major engineering challenges and leading to an understanding of the factors effecting module performance. Each of the 60 modules in the Flight Mirror Assembly (FMA) supports 200-300 densely packed $0.4 \mathrm{~mm}$ thick glass mirror segments in order to meet the unprecedented effective area required to achieve the scientific objectives of the mission. Detailed Finite Element Analysis (FEA), materials testing, and environmental testing have been completed to ensure the modules can be successfully launched. Resulting stress margins are positive based on detailed FEA, a large factor of safety, and a design strength determined by robust characterization of the glass properties. FEA correlates well with the results of the successful modal, vibration, and acoustic environmental tests. Deformation of the module due to on-orbit thermal conditions is also a major design driver. A preliminary thermal control system has been designed and the sensitivity of module optical performance to various thermal loads has been determined using optomechanical analysis methods developed for this unique assembly. This design and analysis furthers the goal of building a module that demonstrates the ability to meet IXO requirements, which is the current focus of the IXO FMA technology development team.
\end{abstract}

Keywords: International X-Ray Observatory, IXO, Module, Flight Mirror Assembly, FMA

\section{INTRODUCTION}

The SXT Modules are the fundamental focusing assemblies of the observatory and development of the technology needed to build the modules is the primary focus of the IXO efforts at NASA Goddard Space Flight Center and the Smithsonian Astrophysical Observatory (SAO). The IXO FMA uses a unique modular design to support the thousands of thin mirror segments needed to achieve the large soft X-ray collecting area of the observatory. Due to the $3.4 \mathrm{~m}$ diameter of the FMA and the need to support $~ 14,000$ segments, a monolithic FMA would be impractical and risky to fabricate and test. Therefore the FMA consists of 60 smaller modules and the primary structure that supports them [1].

An unprecedented combination of soft X-ray collecting area and angular resolution are required by the science objectives for the mission [2]. Where the Chandra X-Ray Observatory had 4 primary/secondary mirror pairs, IXO must have $\sim 360$. Consequently, the mirrors must be much thinner to accommodate the mass and volume constraints of existing launch vehicles. Supporting this large number of very thin mirrors without distorting them at the sub-micron level is the central challenge of the module design.

This paper describes the design and analysis of the modules, a unique optical system needed to advance X-ray astronomy. The design was pursued to a level of detail commensurate with the pre-Phase A IXO mission study including design trade studies, CAD modeling, Finite Element Analysis (FEA), preliminary material selection, thermal analysis, and $\mathrm{X}$-ray performance sensitivity analysis. In some cases the design was developed past what one would expect at this early stage, particularly with respect to optomechanical and structural analysis of the glass mirror segments in order to mitigate perceived mission risks.

*ryan.s.mcclelland@nasa.gov; phone 1301 286-8615 
Table 1. FMA driving requirements.

\begin{tabular}{|c|c|}
\hline Requirement & Value \\
\hline Effective Area & $\begin{array}{c}3.0 \mathrm{~m}^{2} @ 1.25 \mathrm{keV} \\
0.6 \mathrm{~m}^{2} @ 6.0 \mathrm{keV} \\
\end{array}$ \\
\hline Angular Resolution & 4.1 arc-sec \\
\hline Focal Length & $20 \mathrm{~m}$ \\
\hline Mass & $1750 \mathrm{~kg}$ \\
\hline First Axial Mode & $35 \mathrm{~Hz}$ \\
\hline First Torsional Mode & $15 \mathrm{~Hz}$ \\
\hline Quasi-static design loads & $7.5 \mathrm{~g}$ lateral $10.5 \mathrm{~g}$ axial \\
\hline
\end{tabular}

To provide a background to the Module design, this paper also encompasses a brief overview of the IXO Mission and spacecraft, a description of the overall FMA design, an overview of the optical design, and a description of the mechanical characteristics of the mirror segments. An alternate design, also modular, based on the same mission requirements, is being developed at the European Space Agency (ESA) using silicon micro-pore mirror technology [3] and is outside the scope of this paper. The FMA also includes a Hard X-Ray Telescope (HXT) based on existing technology [4] that is not described in detail in this paper.

\subsection{IXO Mission Overview}

The International X-ray Observatory (IXO), planned to launch in 2021, combines elements from NASA's prior Constellation X program and ESA's XEUS program through a collaboration between NASA, ESA, and JAXA. IXO will be a Great Observatory-class mission which builds upon the legacies of the Chandra and XMM-Newton X-ray observatories. IXO will have a mass of around $6600 \mathrm{~kg}$ and will be approximately 23 meters long when deployed and 4 meters in diameter [5]. It will fly on an Atlas 5 or an Ariane V rocket into an L2 halo orbit. The observatory is divided into four spacecraft modules to simplify integration and testing of the observatory as shown in Figure 1. The FMA is contained within the Optics Module mounted near the separation plane with the launch vehicle.

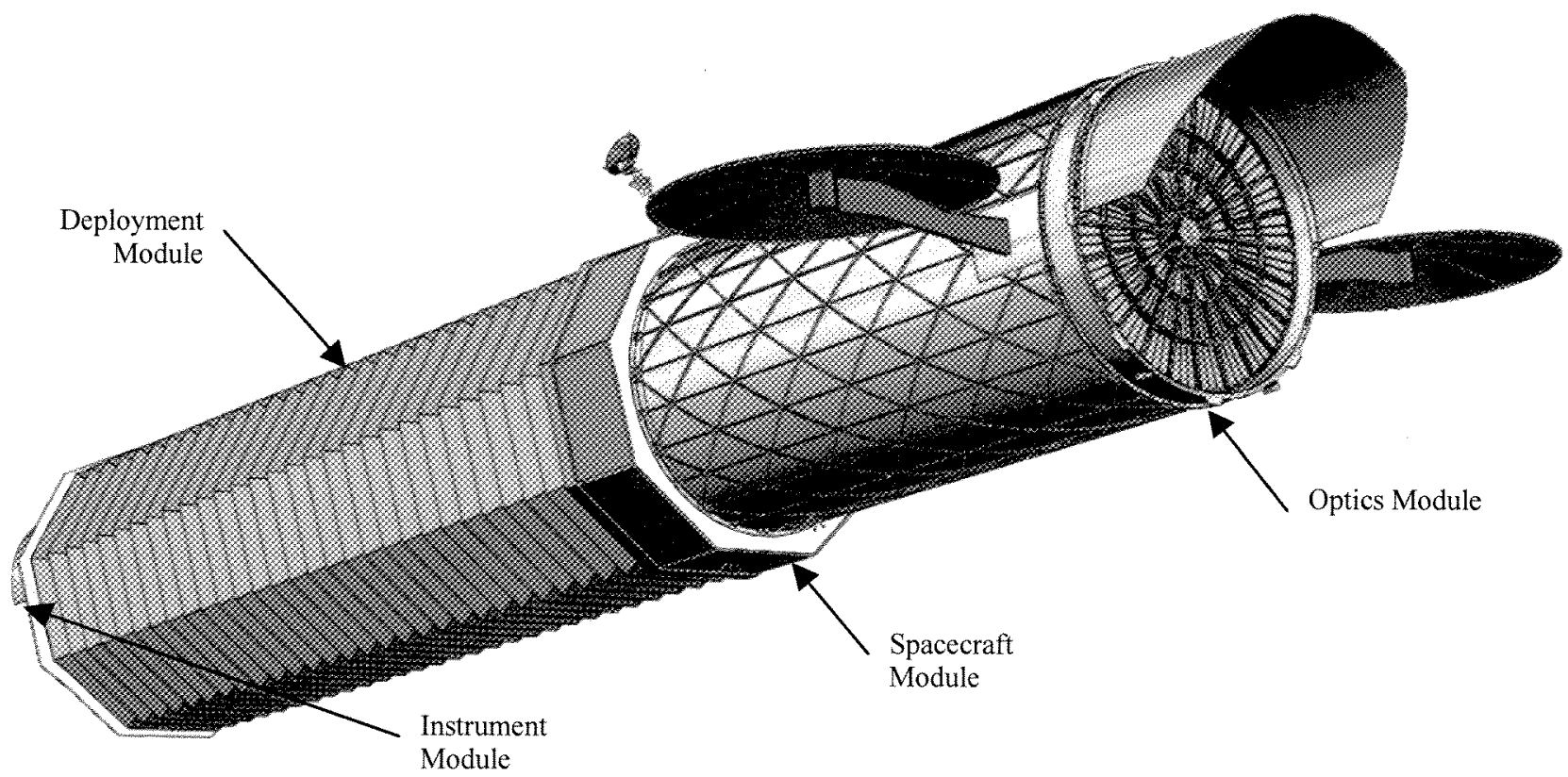

Figure 1. IXO Spacecraft shown in deployed configuration. The assembly is divided into four modules. 


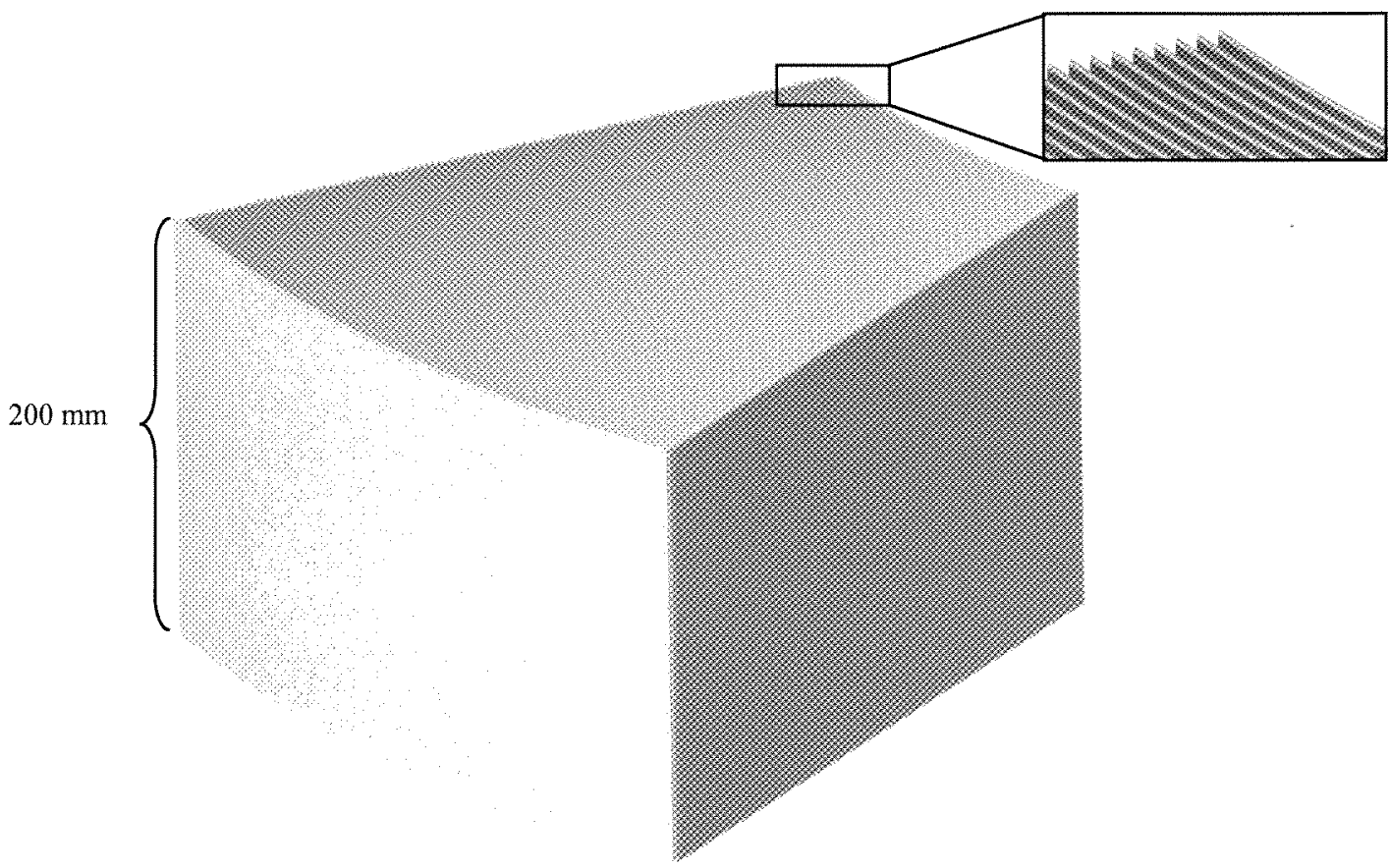

Figure 2. Mirror segments in the inner module demonstrating dense packing of segments. Each model has $200-300$ pairs of mirror segments

\subsection{Segment Characteristics}

The X-ray focusing elements of the modules are the mirror segments. In order to maximize effective area, the segments must be packed together as densely as possible without one primary segment shadowing the next as shown in Figure 2. The thinner the mirror, the more densely the shells can be packed. In order to achieve the effective area requirements shown in Table 1, a large mirror assembly consisting of approximately 360 concentric rings (also called shells) of primary and secondary mirrors was baselined. The diameter of the innermost shell is $744 \mathrm{~mm}$ and that of the outer shell is $3200 \mathrm{~mm}$. A mirror segment thickness of $0.4 \mathrm{~mm}$ allows for sufficient stiffness and strength to support installation and launch while still meeting the effective area and mass requirements. The spacing between mirrors ranges from $1.5 \mathrm{~mm}$ to $4.5 \mathrm{~mm}$.

Segments are slumped from commercially available Schott D263 glass onto polished mandrels to facilitate large scale production [6]. Flat glass sheets are heated in an oven in order to replicate the shape of the forming mandrel at the nanometer level. Each segment is $200 \mathrm{~mm}$ in axial length and $167-392 \mathrm{~mm}$ in circumferential span. The total mass of all 13,896 glass segments in the FMA is $730 \mathrm{~kg}$.

\subsection{FMA Design Overview}

The FMA consists of 60 SXT modules, each containing approximately 200-300 mirror segments, mounted into the FMA primary structure as shown in Figure 3. The inner ring has 12 modules and the middle and outer ring have 24 modules each. The Hard X-ray Mirror Module (HXMM) is mounted into a central hole in the primary structure. Note that the term 'module' in this paper generally refers to the SXT modules rather than the HXMM. The FMA mounts to a spacecraft adapter ring via 24 bolted interfaces located around the perimeter of the primary structure. 


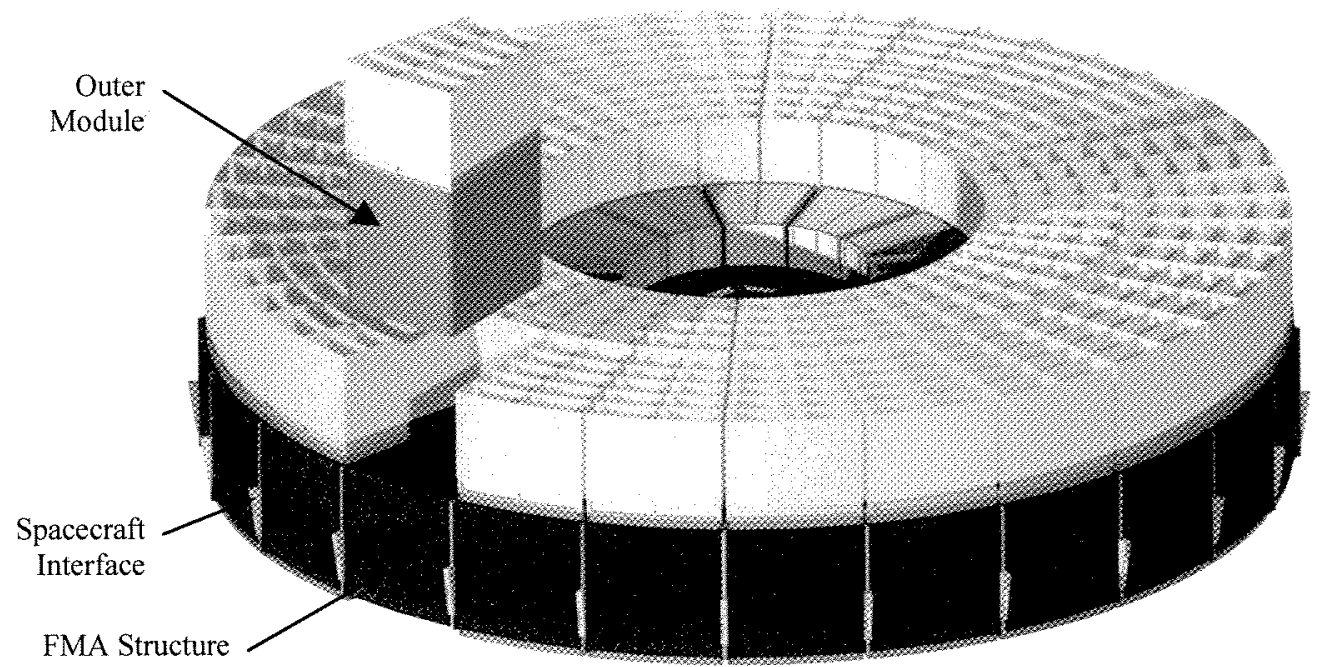

Figure 3. Exploded view of FMA illustrating the basic parts of the assembly.

\section{MODULE DESIGN}

The modules consist of optical, structural, and thermal elements including 200-300 primary and secondary mirror segments, tabs for bonding the mirrors to the module, structural rails and panels, kinematic mounts to attach to the FMA primary structure, a stray light baffle, and a thermal pre-collimator as shown in Figure 4. These elements facilitate integration of the mirror segments, launching of the modules within the FMA, and thermal control during on-orbit operation. The following sections detail the functionality, design, and analysis of these elements. The continued development of the module and associated mirror technologies will lead to the fabrication and testing of an engineering unit which will demonstrate the design's capability to meet IXO requirements.
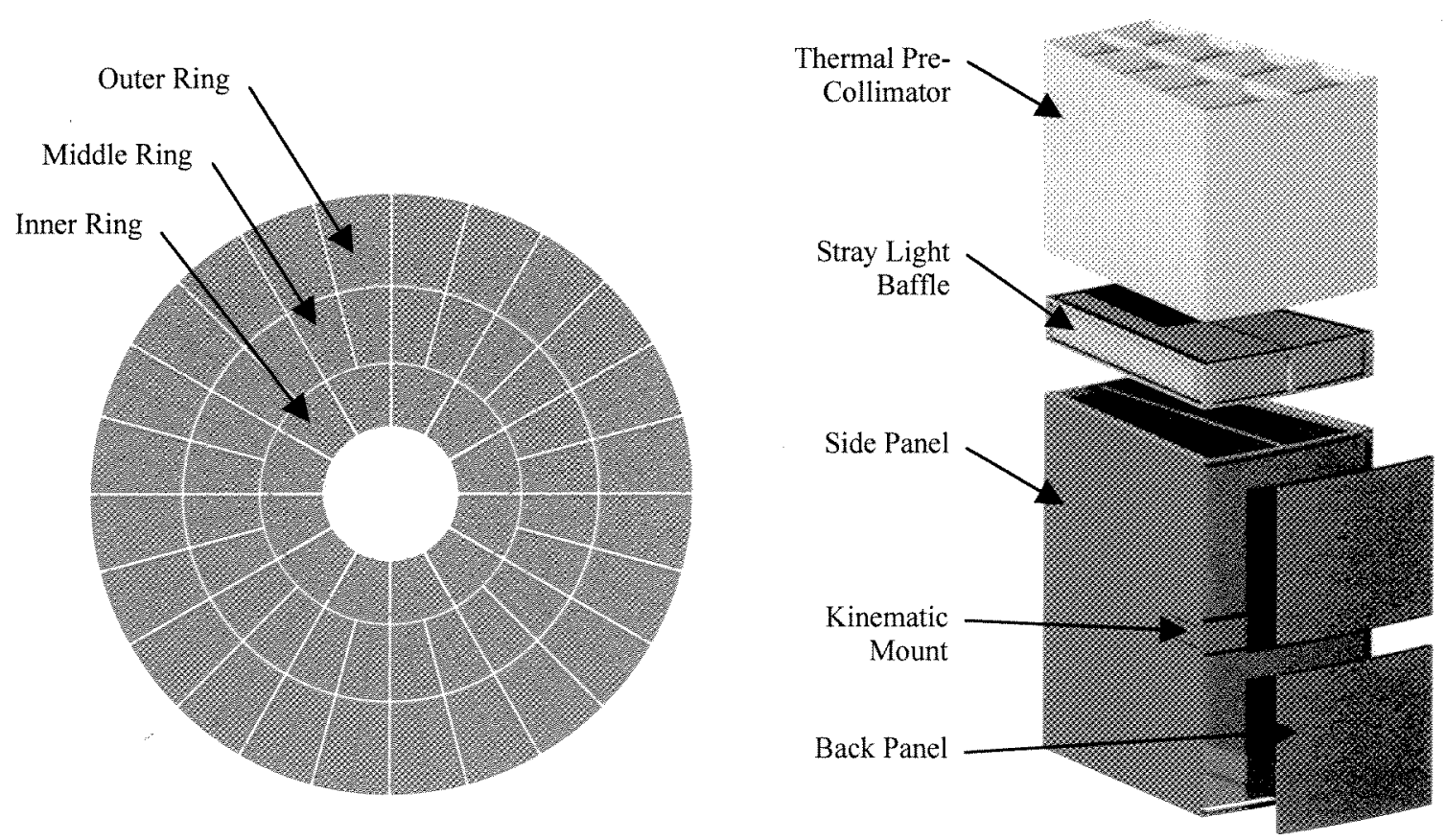

Figure 4. Top view of FMA with primary structure hidden illustrating the12/24/24 module layout (left) and exploded view of a middle ring module (right). 


\subsection{Module Size and Layout}

The module design is strongly affected by the layout and sizing of the modules. The maximum circumferential size of a module is limited by the mirror fabrication process to $400 \mathrm{~mm}$ (maximum segment size). Based on this dimension and the outer diameter of the FMA, a minimum of 24 outer modules are needed. In order to maintain a size and mass amenable to handling, the FMA is divided radially into three rings of modules as shown in Figure 4 . The inner ring is divided into 12 modules due to the mirror size limitation while the middle and outer rings are divided into 24 modules due to a combination of the mirror size limitation and the desire to maintain 12-fold symmetry. Since the degree of symmetry between the module rings determines where primary structure can exist, the design of the primary structure is constrained by the module layout. The 12-fold symmetry allows an efficient design of the primary structure so that each module has a direct load path to the spacecraft interface. The 12/24/24 module layout leads to 60 total modules.

The necessary effective area could also be achieved by more numerous but smaller modules. However, it is desirable to minimize the module count in order to reduce the testing time, since each module will go through an extensive acceptance testing program.

\subsection{Module Structural Design}

The module assembly includes the mirror segments and the supporting structure as well as additional thermal and optical elements. The structure consists primarily of load bearing panels which close out the module on all but the axial ends, which must remain open to allow X-rays to pass through. The panels also project the segments from direct impingement of acoustic energy and Foreign Object Damage (FOD).

The mirrors are mounted to the module structural panels at eight points: three along each side edge and one along each top and bottom edge as shown in Figure 7. This bond configuration is necessary both to prevent unacceptable distortion in gravity and reduce the stress in the glass. At each mounting location, a rail is fastened to the module structure as shown in Figure 5. During integration, tabs are bonded to the rail before each mirror is installed then the mirrors are bonded to the tabs using UV cure adhesive for a near-instantaneous cure [7].

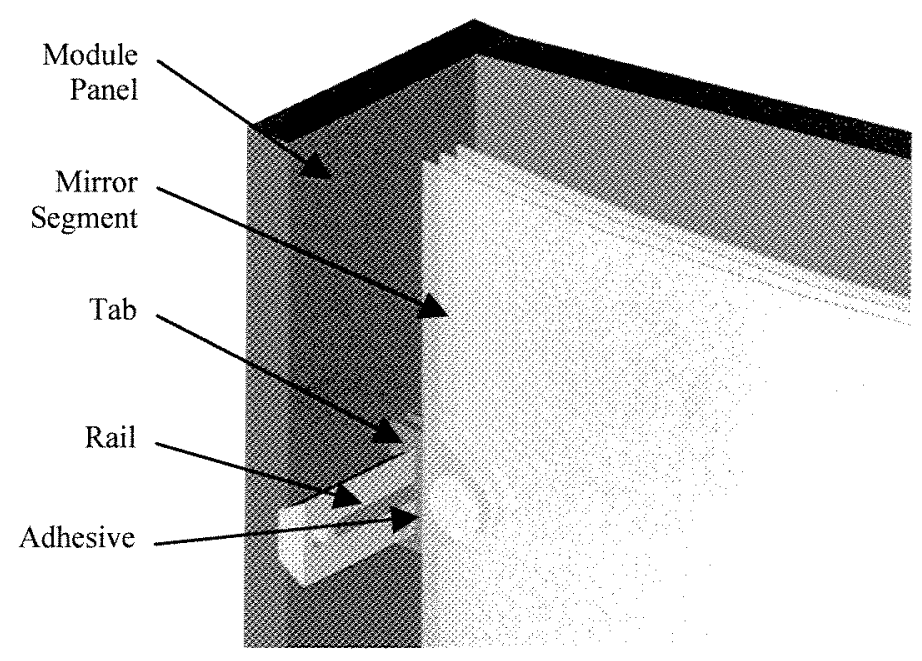

Figure 5. Detail of a demonstration module showing attachment of mirror segments to module structure.

The material used for the module structure must have a Coefficient of Thermal Expansion (CTE) closely matching the $6.3 \mathrm{ppm} / \mathrm{C}$ CTE of the D263 glass segments. A high modulus to density ratio (stiffness to weight ratio), high thermal conductivity, and good machinability are also desired. Kovar, a Nickel/Iron alloy, was selected as the baseline material due to its closely matching CTE of $6.7 \mathrm{ppm} / \mathrm{C}$. Other materials being considered include Carbon Fiber Reinforced Plastic (CFRP), Titanium alloys, and various metal matrix composites. 


\section{MIRROR STRUCTURAL ANALYSIS AND TESTING}

Of particular concern in the structural analysis of the FMA and modules is the stress experienced by the glass segments during launch. In order to develop a Margin of Safety for the mirror segments, the Ultimate Tensile Strength, appropriate Factor of Safety, quasi-static design loads, and maximum glass stress must be determined.

\subsection{Statistical Glass Strength Determination}

Determining the strength of glass is more complex than for an analogous metal optic due to the nature of brittle glass failure, which is dependent on the size and distribution of surface flaws. The statistical strength of a population of glass segments is effectively expressed by the two parameter Weibull distributions which describes the Probability of Failure (POF) as a function of the characteristic strength $\left(\_0\right)$ and the Weibull modulus $(\mathrm{m})$ [8].

$$
P O F=1-\exp \left[-\left(\frac{\sigma}{\sigma_{0}}\right)^{m}\right]
$$

The strength of a test specimen can be related to the strength of a glass segment as supported in the FMA, which have different stressed areas and therefore a different number of flaws, by the following equation [9]:

$$
\frac{\sigma_{1}}{\sigma_{2}}=\left(\frac{A_{2}}{A_{1}}\right)^{\frac{1}{m}}
$$

Extensive strength tests, including both folding tests and tests simulating the segment-to-module bonding geometry, have been performed on slumped glass segments in order to determine the Weibull parameters. Results from the tests using a simulated bond joint, scaled by the number of bond areas, are used for strength calculations due to their superior representation of the actual stress state.

\subsection{Ultimate Tensile Strength}

Options for determining the Ultimate Tensile Strength (UTS) for the design of glass parts are detailed in NASA-STD5001. One option is to use the POF, based on the desired reliability of the system and the number of glass segments, to calculate the UTS from the Weibull parameters and equation (1). For instance, selecting a 1 in 100 POF for each segment would necessitate a 1 in 1.4 million POF for the entire FMA, due to the 14,000 segments, which yields an UTS of only $10 \mathrm{MPa}$. Using this method, one must design for the weakest segment statistically possible.

Alternately, each mirror segment may be proof tested to the desired UTS in order to screen out segments with insufficient strength. Using the proof test option, the UTS used is only limited by the number of segments it is acceptable to fail during proof testing. For instance, using a strength of $40 \mathrm{MPa}$ would yield a POF of 1 in 1000, so one would only expect one segment in 1000 to fail the proof test (see Figure 6). Based on the proof test option, an UTS of $40 \mathrm{MPa}$ is used for margin calculations throughout in this paper. 


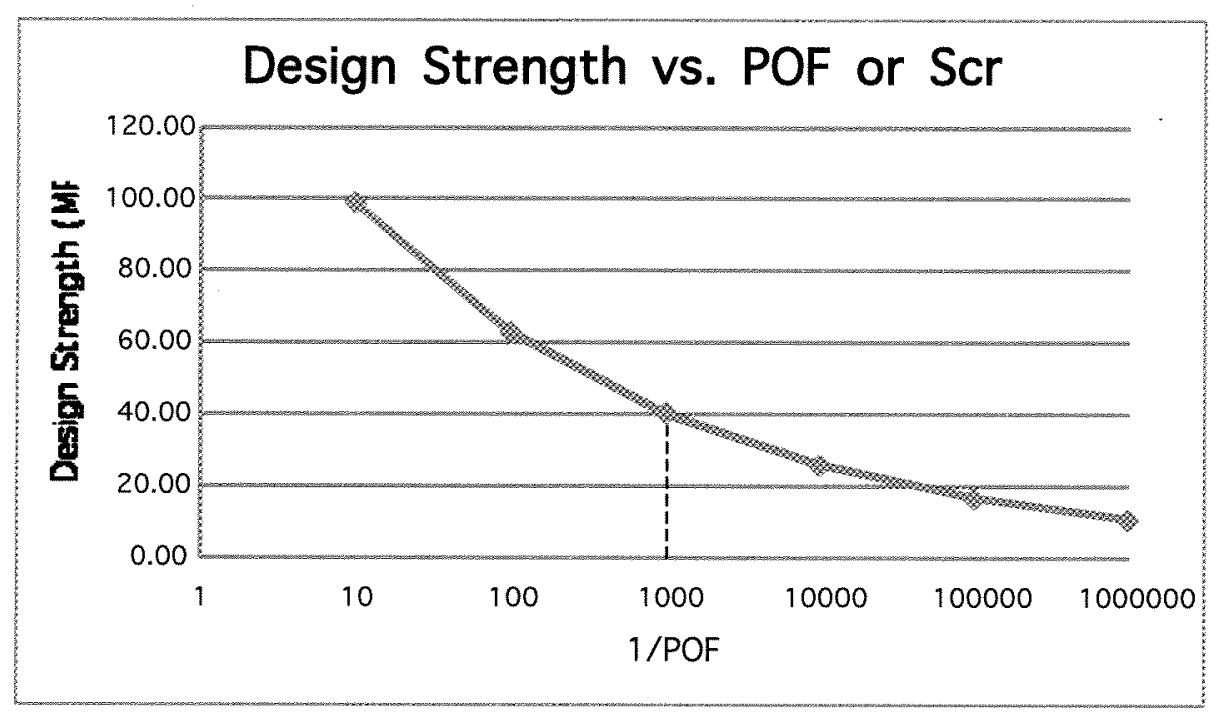

Figure 6. Design strength as a function of POF based on Weibull parameters from materials testing.

\subsection{Quasi-static Design Loads}

In order to take into account the effect of the vibration response of the FMA primary structure and IXO observatory on the module loads, a sine response analysis was performed on an integrated FEM of the observatory. A sine sweep in each axis was input at the base of the stowed observatory model and net center of gravity (CG) accelerations were recovered at the spacecraft, FMA, and module levels. The amplitude of the sine sweep was based on the Atlas mission planner's guide. The resulting maximum accelerations for the inner module (worst case) were determined to be $8.5 \mathrm{~g}$ lateral and $18 \mathrm{~g}$ axial. These accelerations were then used as quasi-static design loads applied to the mirror segment FEM as described in the following section. Additional loads refinement will occur in later project phases when a true Coupled Loads Analysis is performed.

\subsection{Glass stress and resulting Margin of Safety}

Detailed solid element FEMs of the worst case segment were used to predict the maximum stress in the glass. The outermost segment of the inner module was chosen due to its large azimuthal span and relatively high curvature. Several bond geometries were investigated including the baseline semi-circular bond with a $3 \mathrm{~mm}$ radius shown in Figure 7 .
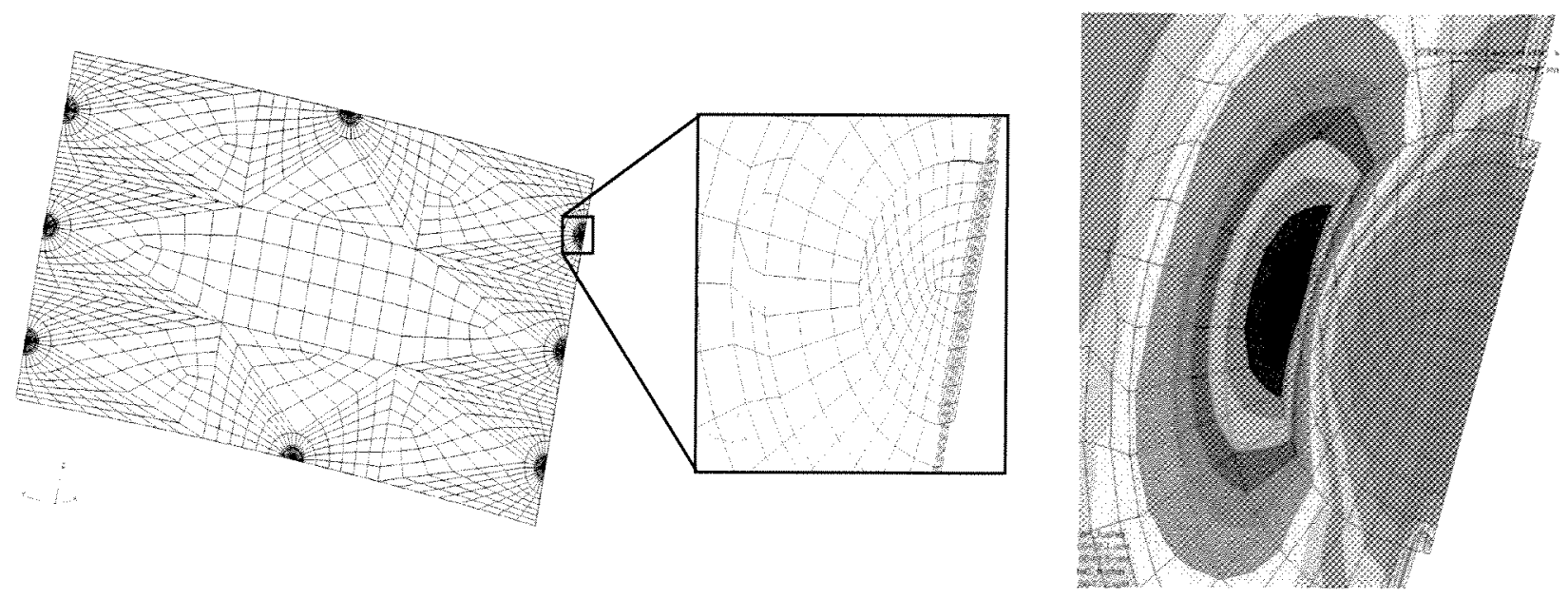

Figure 7. Detailed FEM of mirror segment used for determination of maximum stress (left) with detail of bonding area and plot showing peak stress location (right). 
Appling the quasi-static design loads to the worst case segment FEM resulted in maximum stress of $3.1 \mathrm{MPa}$. The peak stresses occur near the bond points as shown in Figure 7. Using a design strength of $40.0 \mathrm{MPa}$ and Factor of Safety of 3.0 as previously described yields a Margin of Safety of 3.3. The maximum principal stress failure criterion was used due to the brittle nature of glass failure. Based on this result, the modules can be successfully launched with a large stress margin and Factor of Safety.

\subsection{Environmental testing}

In order to ensure that the behavior and strength of the glass is well understood when subjected to the launch environment, a series of environmental tests were performed on segments in a simulated module structure. The test series included static load testing, modal tap testing, random vibration testing, acoustic testing, and shock testing. The acoustic test was successfully performed at the full Altas 551 qualification level, demonstrating a low sensitivity to acoustic loads. The shock test was performed until failure. The highest acceleration sustained without failure (as measured at the mirror bonding rail) as $3000 \mathrm{~g}$. The shock sensitivity of the segments will be taken into consideration when selecting spacecraft deployment mechanisms.

In general, segment response including modes and stresses correlated well with analysis predictions. Pre- and post-test mirror figure measurements show the mirror figure did not change as a result of environmental tests.
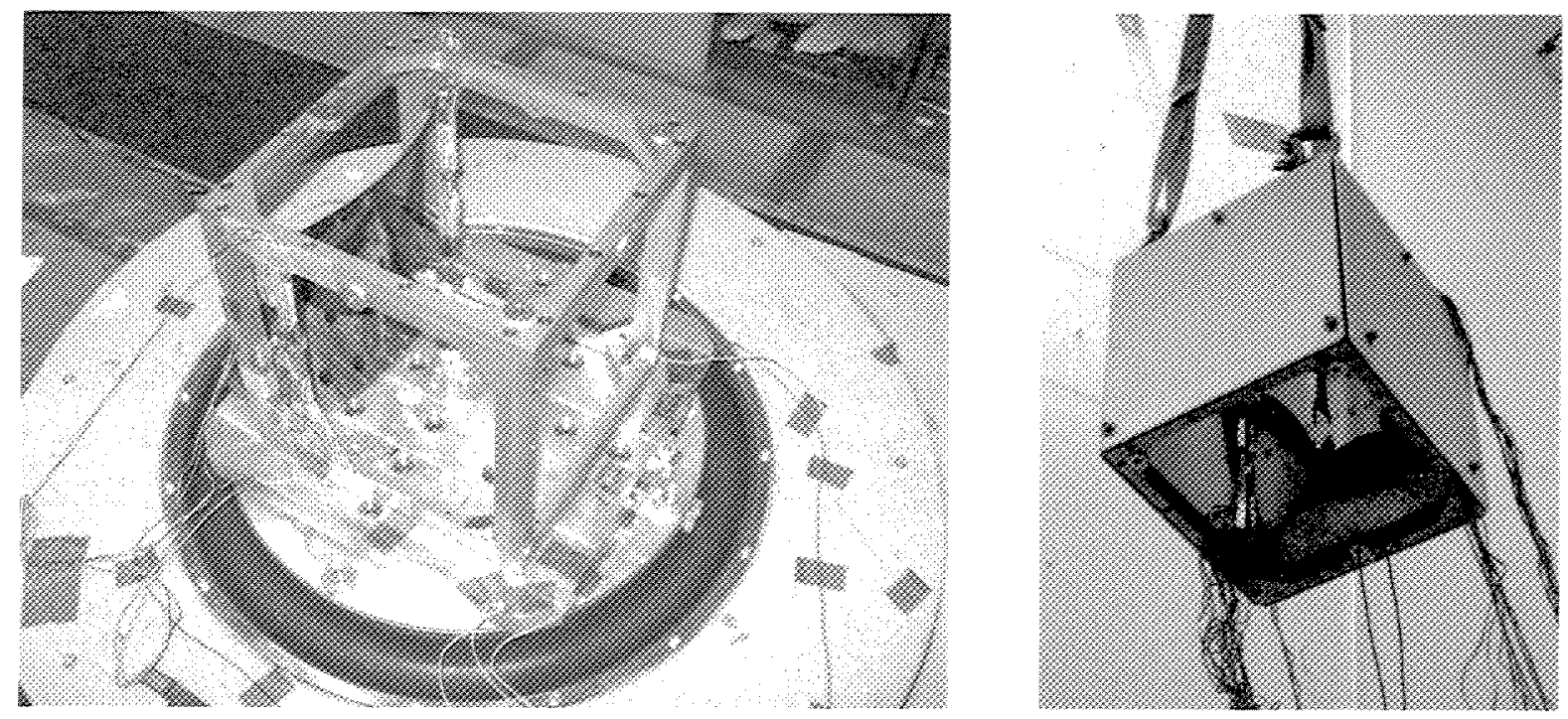

Figure 8. Testing of three mirror simulated module. Shock test configuration (left) and acoustic test configuration (right).

\section{THERMAL DESIGN AND OPTOMECHANICAL ANALYSIS}

\subsection{Thermal Design}

The module will be kept near room temperature during both ground integration and on-orbit operation in order to prevent unacceptable deformations due to thermal distortion. A Half Power Diameter (HPD) error of 1.0 arc-seconds has been allocated in the error budget for thermal effects. Thermal control is aided by the relatively quiescent L2 thermal environment and limitations on the roll and yaw of the observatory $\left( \pm 20^{\circ}\right.$ yaw, $\pm 10^{\circ}$ roll). A sunshade will prevent direct illumination of the Modules. The challenge of the thermal system design is to replace the heat lost to space by the segments while minimizing the thermal gradients over the modules.

Heat lost to space is replaced by heaters on the Stray Light Baffles (SLBs) and the forward two meters of the metering structure as shown in Figure 9. Multiple heater zones are actively controlled to minimize the thermal gradients and bulk temperature changes in the modules. Optically, the SLBs serve to prevent stray X-rays from focusing to the image plane after only a single bounce off a primary or secondary mirror. The SLBs consist of a series of aluminum vanes placed 
directly above each pair of mirrors, providing a convenient structure to which heat can be applied to the segments with good radiative coupling.

On the inner modules, reduction of heat lost to space by the segments is provided by thin thermal covers mounted on the axial ends of the modules. The higher energy X-rays focused by the inner modules can pass through the thermal covers due to their greater penetration capability. On the middle and outer modules, which focus lower energy X-rays, the heat lost to space is reduced by G10 thermal pre-collimators which reduce the view of the segments to space without blocking the module aperture, as shown in Figure 9. Based on this design, approximately $1500 \mathrm{~W}$ of heater power is required to keep the modules at room temperature in the coldest case [10].
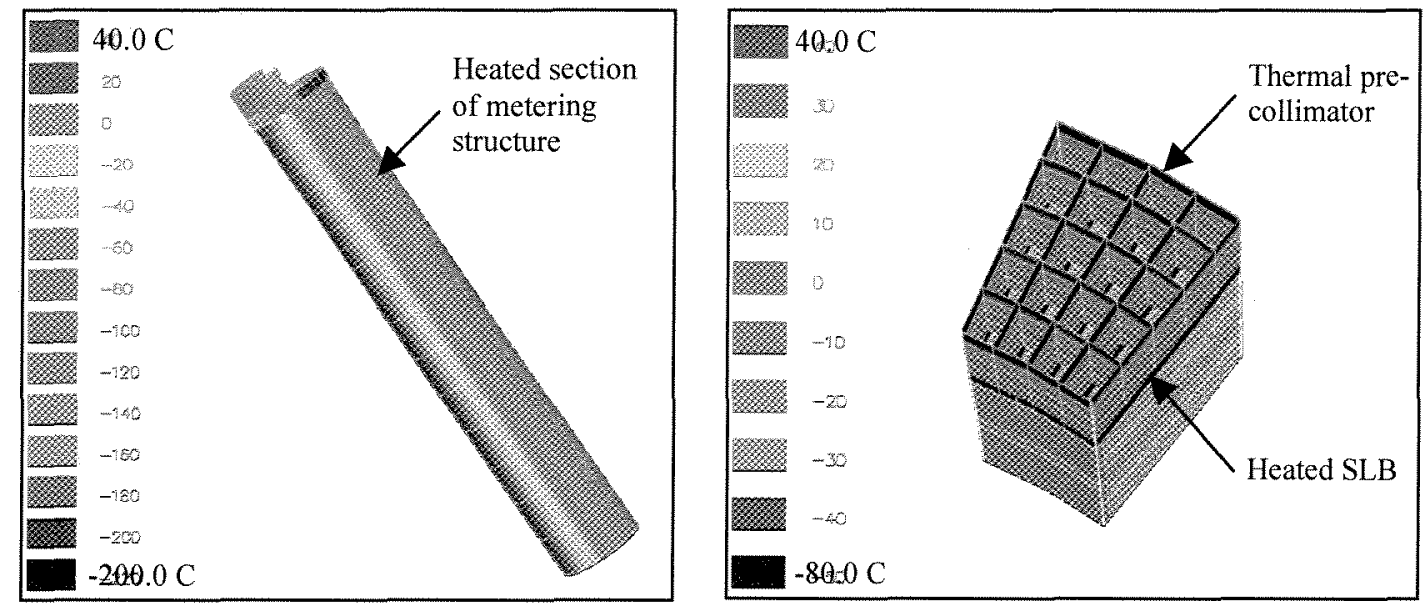

Figure 9. Thermal model of IXO Spacecraft showing heated section of metering structure (left) and predicted module temperatures (right).

\subsection{Optomechanical Sensitivity Analysis}

A detailed Finite Element Model (FEM) of an inner module was generated in order to determine the performance of the module design under various thermal and structural loads (Figure 10). Generating the hundreds of unique mirror segment FEMs within the module with sufficient accuracy and element density to allow for ray-traced X-ray performance prediction was particularly challenging. Custom software was written to allow the segment FEMs to be automatically generated with the desired mesh density based on the optical prescription file. Additional custom software was written to extract the FEA output and ray-trace the results to generate performance predictions for both individual segments and entire modules. Performance predictions are based on the low order surface deformations and equations published in reference [11]. 

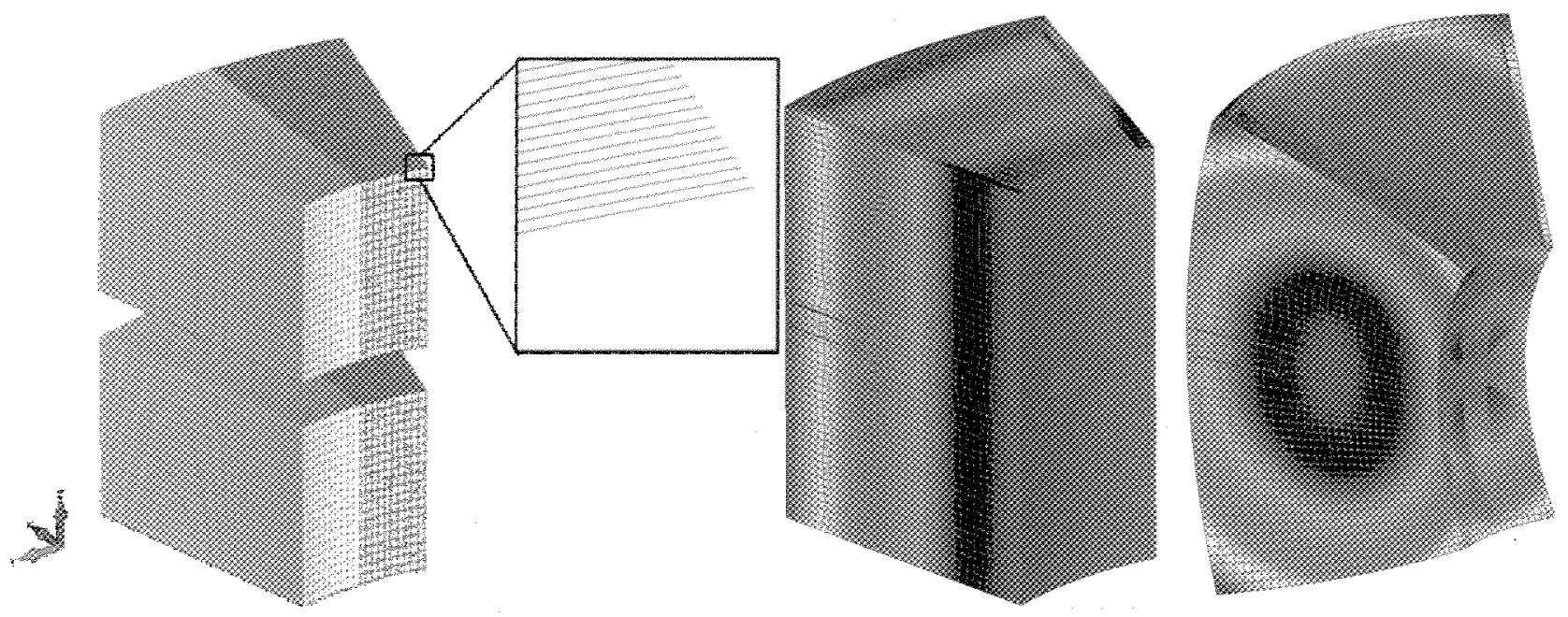

Figure 10. Software generated FEM of an inner module with each mirror segment represented (left). Radial temperature gradient applied to model (center) and resulting deformations (right). Colors indicate temperature (center) and displacement (right).

In order to direct the module structural and thermal designs, the sensitivity of X-ray performance was predicted with respect to various design parameters and thermal loads as shown in Table 2. The HPD error scales linearly with both the bulk temperature change and CTE mismatch between the glass segments and structure (cases 1-3). Based on this sensitivity analysis, it can be calculated that for the expected bulk temperature change of $\pm 0.1^{\circ} \mathrm{C}$ a CTE match of $3.3 \mathrm{ppm} /{ }^{\circ} \mathrm{C}$ or better is needed to limit the HPD error to 1.0 arc-second, suggesting the structural material must have a CTE between 3.0 and $9.6 \mathrm{ppm} /{ }^{\circ} \mathrm{C}$.

Table 2. Predicted sensitivity of module X-ray HPD performance to various thermal cases.

\begin{tabular}{|c|l|c|}
\hline Case \# & \multicolumn{1}{|c|}{ Description } & HPD \\
\hline 1 & $1^{\circ} \mathrm{C}$ Bulk Temp 0.0ppm/C CTE Mismatch 0.0 \\
\hline 2 & $1^{\circ} \mathrm{C}$ Bulk Temp 1.0ppm/C CTE Mismatch 3.0 \\
\hline 3 & $1^{\circ} \mathrm{C}$ Bulk Temp 2.0ppm/C CTE Mismatch 6.0 \\
\hline 4 & $1^{\circ} \mathrm{C}$ x (radial) Gradient & 1.6 \\
\hline 5 & $1^{\circ} \mathrm{C}$ y (tangential) Gradient & 0.3 \\
\hline 6 & $1^{\circ} \mathrm{C}$ z (axial) Gradient & 0.1 \\
\hline 7 & $1^{\circ} \mathrm{C}$ Temp Gradient Structure to Segment 9.5 \\
\hline 8 & $2^{\circ} \mathrm{C}$ Temp Gradient Structure to SegmenBs8.9 \\
\hline 9 & $1^{\circ} \mathrm{C}$ Bulk Temp with Flexures & 2.6 \\
\hline 10 & $1^{\circ} \mathrm{C}$ Bulk Temp with Half Thickness Flexures5 \\
\hline
\end{tabular}

It can be seen in Table 3, cases 4-6, that the HPD error is relatively insensitive to thermal gradients in the $z$ (axial) and $y$ (tangential) directions compared with the $\mathrm{x}$ (radial) direction. This is expected because a radial gradient causes a misalignment between the primary and secondary segments, to which the HPD error is very sensitive (see Figure 10).

The most sensitive case run was for a temperature gradient between the glass mirrors and module structure (cases 7 and 8, CTE matched materials assumed). Heating the module structure was proposed as a method of replacing heat lost by the mirrors to space. It can be seen from the sensitivity analysis that this is not a viable option since this gradient must be kept within $0.05^{\circ} \mathrm{C}$ to meet the 1.0 arc-second error allocated to thermal effects.

In the above cases, the flexures mounting the module to the FMA were assumed to be perfectly kinematic by the use of idealized FEM constraints. The effect of non-idealized flexures can be seen in Table 3, cases 9 and 10 . Halving the 
thickness of the flexures reduces the HPD error by a factor of 5, indicating the HPD is very sensitive to the flexure design. Optimization of the flexure design is expected to be an area of significant work in the future.

These results were taken into account in material selection and thermal design of the module. Full mapping of the predicted module temperatures to the FEM and subsequent optomechanical analysis will take place in a later project phase.

\section{CONCLUSIONS}

Preliminary design and analysis of the mirror modules has been completed to a level commensurate with the Pre-Phase A mission study for which the baseline design was developed. Detailed structural analysis of the thin glass mirror segments has been performed and demonstrates the compatibility of the module design with the launch loads. Environmental tests have been performed to correlate the FEA and verify the strength of the mirrors. Methods for predicting mirror figure errors based on FEA distortions have been developed and used to perform sensitivity analysis based on thermal loads that drive the thermal and mechanical design. Future work generally involves adding detail to the design and analysis including the module panels, structural connections, kinematic mounts, thermal components, and optomechanical analysis. The end goal is to build a module prototype demonstrating the ability to meet the IXO FMA requirements.

\section{REFERENCES}

[1] R. McClelland and D. Robinson, "Design Concept for the International X-Ray Observatory Flight Mirror Assembly" 2009 IEEE Aerospace Proceedings, March 6-13, 2009.

[2] J. Bookbinder, R. Smith, A. Hornschemeier, et al, "The Constellation-X Observatory" Proc. of SPIE Vol. 7011, 701102, 2008.

[3] K. Wallace, M. Bavdaz, P. Gordon, "Silicon pore optics development” Proc. Of SPIE, 7437 (2009).

[4] J. Koglin, F. Christensen, W. Craig, et al, "NuSTAR hard X-ray optics" Proc. Of SPIE 5488, 856 (2004).

[5] D. Robinson and R. McClelland, "Mechanical overview of the International X-Ray Observatory" 2009 IEEE Aerospace Proceeding, March 6-13 (2009).

[6] W. Zhang, K. Chan, G. Byron, et al, "Mirror technology development for the International X-ray Observatory mission" Proc. Of SPIE, 7437 (2009).

[7] T. Evans and T. Saha, "Arc Second Alignment of International X-Ray Observatory Mirror Segments in a Fixed Structure" 2010 IEEE Aerospace Proceeding, March 6-13 (2010).

[8]A. F. McLean, "An overview of the ceramic design process" Engineered Material Handbook: Ceramics and Glasses Materials Park, OH: ASM International (1991), vol. 4, pp. 676-689

[9] K. B. Doyle and M. A. Kahan, "Design strength of optical glass" Proc. of SPIE Vol. 5176 (2003)

[10] M. K. Choi, "Thermal Considerations for Meeting $20^{\circ} \mathrm{C}$ and Stringent Temperature Gradient Requirements of IXO SXT Mirror Modules" SAE 2009-01-2391 (2009).

[11] T. Saha, "Image defects from surface and alignment errors in grazing incidence telescopes" Opt. Eng. 29, 1296-1305 (1990). 